\title{
Monophasic Action Potential Duration During Programmed Electrical Stimulation
}

\author{
NABIL KANAAN, JANICE JENKINS, KEITH CHILDS, YU-ZHI GE, and \\ ALAN KADISH \\ From the Bioengineering Program, Department of Electrical Engineering and Computer Science \\ and the Department of Internal Medicine, The University of Michigan, Ann Arbor, Michigan, \\ and the Division of Cardiology, Department of Internal Medicine, Northwestern University, \\ Chicago, Illinois
}

\begin{abstract}
KANAAN, N., ET AL.: Monophasic Action Potential Duration During Programmed Electrical Stimulation. To examine changes in monophasic action potential duration (APD) with a pacing protocol similar to that used during electrophysiological testing, action potentials were recorded in vivo from the left ventricular apical endocardium of 12 normal mongrel dogs. The atrioventricular node was ablated and the dogs paced from the anterior right ventricle at a baseline cycle length of $1000 \mathrm{~ms}$ between interventions. Mean steady-state APD (APD ${ }_{\mathrm{ss}}$ ) was $266 \pm 7 \mathrm{~ms}$ at a pacing cycle length (PCL) of $1000 \mathrm{~ms}$. Two pacing protocols were used. The first consisted of a sudden acceleration in pacing from a cycle length of 1000 $\mathrm{ms}$ to one between 300 and $600 \mathrm{~ms}$. The second consisted of an 8-beat train at a cycle length of $400 \mathrm{~ms}$ followed by a premature beat at a coupling interval of $280 \mathrm{~ms}$ followed by a pause. The inter-train pause varied between 1 second and 32 seconds. With a sudden acceleration in pacing rate, steady-state values for APD at the faster PCLs were significantly smaller than APD ${ }_{\mathrm{ss}}$ at $1000 \mathrm{~ms}$ with a change to cycle lengths of $600 \mathrm{~ms}(247 \pm 29 \mathrm{~ms}), 500 \mathrm{~ms}(229 \pm 21 \mathrm{~ms}), 400 \mathrm{~ms}(220 \pm 17 \mathrm{~ms})$, and $300 \mathrm{~ms}(203 \pm 31$ ms; $P<0.01$ for all comparisons). The time constant of the change in APD was shorter at a PCL of 300 $\mathrm{ms}(14.9 \pm 0.8 \mathrm{~s})$ than $600 \mathrm{~ms}(20.3 \pm 4.7 \mathrm{~s} ; \mathrm{P}<0.05)$. With drive train pacing and incorporating an inter-train pause, the percent drop in steady-state APD compared to APD for the first train ranged from $10.1 \%$ with a 1 -second inter-train pause to $2.1 \%$ with a 32 -second pause. The difference in APD between the first drive train and drive trains after at least 3 minutes of pacing when APD had stabilized was not significant for an inter-train pause exceeding 8 seconds. In conclusion: (1) with a sudden acceleration in pacing rate, endocardial APD in vivo decreases exponentially. The faster the new rate, the shorter the new steady-state APD and the shorter the time constant. (2) When pacing using an 8-beat drive train and an inter-train pause, there is a decremental shortening in APD for pause lengths shorter than 16 seconds. Thus, while performing programmed stimulation using a pause, a conditioning period of at least 2 minutes should be used prior to diastole scanning to allow APD to achieve a steady state. (PACE, Vol. 14, June 1991)
\end{abstract}

monophasic action potential, action potential duration, programmed electrical stimulation, time constant

Supported in part by NIH Grant HL 40667.

Dr Kadish is a scholar of the February Cardiovascular Research Institute

Address for reprints: Alan Kadish, M.D., Northwestern Memorial Hospital, 250 E. Superior Suite 524, Chicago, IL 60611. Fax: (312) 908-6003.

Received October 4, 1990; revision January 29, 1991; accepted March 12, 1991.

\section{Introduction}

Action potential duration (APD) in cardiac muscle is inversely proportional to the heart rate. ${ }^{1-3}$ When the rate of stimulation is suddenly increased, each succeeding action potential is further shortened over a period of several minutes until a steady-state APD is reached. ${ }^{4}$ This steady- 
state APD is characteristic of the frequency of stimulation and is directly related to the diastolic interval. ${ }^{5}$ During clinical or experimental studies, a stimulation protocol consisting of 8 stimuli at twice diastolic threshold $\left(8 \mathrm{~S}_{1}\right)$ followed by one or more extrastimuli and a pause between drive trains is frequently used to study the refractory period in the ventricles and to perform programmed electrical stimulation. Based on the previous observations, it would be predicted that the steady-state APD after several drive trains will depend on the inter-train pause length; the longer the pause, the lesser the cumulative effect of successive drive trains. However, APDs during programmed stimulation have not been well characterized.

The purpose of this study was to examine APD during programmed stimulation and to compare the steady-state APD during constant cycle length pacing to pacing with an inter-train pause with a stimulation protocol similar to that used in electrophysiology studies.

\section{Methods and Materials}

\section{Experimental Set-Up}

Experiments were performed on 12 mongrel dogs weighing between 15 and $30 \mathrm{~kg}$. The animals were anesthetized with pentobarbital administered intravenously, intubated with an endotracheal tube and ventilated with room air using a volume ventilator. The chest was opened with a mid-sternal incision and the pericardium incised to expose the heart. Fifty joule shocks were delivered between the aorta and the tricuspid valve to ablate the atrioventricular node in order to allow for external control of ventricular rate. Pacing was performed at twice diastolic threshold from the right ventricle using a bipolar electrode at rates varying between 300 and $1000 \mathrm{~ms}$.

Monophasic action potentials (MAPs) were recorded from the left ventricular endocardium using a contact electrode. ${ }^{6.11}$ Contact action potential recording, which has been shown to provide a useful insight into some important electrophysiological properties of myocardial cells, ${ }^{6-11}$ is easier to obtain than intracellular recording. Furthermore, it is less prone to cause irreversible tissue injury than recording with suction electrodes. ${ }^{9}$

Electrocardiographic (ECG) surface leads and endocardial MAPs were simultaneously amplified and displayed on a multichannel oscillographic recorder (Model VR-12, Electronics for Medicine, Pleasantville, NY, USA) with differential input impedance of $1 \mathrm{M} \Omega$ and a frequency response of 0.05 to $500 \mathrm{~Hz}$. Endocardial signals were also recorded on FM magnetic tape (Model 3968A, Hewlett Packard, Andover, MA, USA) with a frequency response of DC to $1250 \mathrm{~Hz}$ at a tape speed of 3 and $3 / 4 \mathrm{in} / \mathrm{s}$ for subsequent playback and offline computer acquisition and analysis.

The digital data acquisition system has been described elsewhere. ${ }^{12}$ Briefly it consisted of a Macintosh II microcomputer with 5 megabytes of memory, a 40 megabyte hard disk, and a 600 megabyte optical drive for permanent data storage. Data acquisition was performed using the MacADIOS plug-in board (GW Instruments, Somerville, MA, USA), a 12-bit analog-to-digital (A/ D) converter with $5-\mu \mathrm{s}$ acquisition time. The MacADIOS memory board is mapped into the Macintosh II memory for direct memory access. Input-output operations can thus be accessed by user-written assembly language or a high level language.

A software slope detector was designed for recognition of individual MAP onsets. The program searches for a location where the slope over a 10-point sliding window exceeds a preset threshold level. Thus a point on the initial upstroke of the action potential is identified as onset of activation. APD was computed using a tangent technique previously developed in our laboratory. ${ }^{12}$ The technique replicates hand measurements of APD, which consisted in manually extending the repolarization phase of the action potential until intersection with the baseline. ${ }^{13-15}$ APD is thus found by measuring the time interval from the onset of activation to the intersection point.

\section{Pacing Protocols}

Two different pacing protocols were used in this study. The first (protocol A) consisted of a sudden acceleration in pacing cycle length (PCL) to a new faster rate $\left(\mathrm{PCL}_{\mathrm{f}}\right)$. Pacing was performed 
for 3 minutes and $\mathrm{PCL}_{\mathrm{f}}$ varied on successive trials from 600 to $300 \mathrm{~ms}$ in $100 \mathrm{~ms}$ decrements. The second (protocol B) consisted of successive trains (8 $\mathrm{S}_{1}$ at $400 \mathrm{~ms}$ followed by a premature at 280 $\mathrm{ms}$ ) separated by an inter-train pause. The pause length was set at $1,2,4,8,16$, and 32 seconds on successive trials. Animals were paced at $1000 \mathrm{~ms}$ during the inter-train pause and data were also acquired for a period of at least 3 minutes. In both protocols, at least 5 minutes were allowed to elapse between interventions to allow a return of APD to baseline. Although APD with a sudden acceleration in pacing rate has been previously studied, the goals of this protocol were to: (a) compare APD during constant cycle length pacing to pacing with an inter-train pause in the same experiments; and to (b) examine the rate of change of APD during a sudden acceleration in stimulation frequency.

\section{Data Analysis}

$\mathrm{APD}_{\mathrm{ss}}$ at $1000 \mathrm{~ms}$ and steady-state APDs for different fast cycle lengths from protocol A were compared using ANOVA. The steady-state APD was obtained by averaging the APD for at least 100 MAPs. In the case of protocol B, APD of the first train and end-of-train APD after 3 minutes of pacing were compared for different pause lengths using ANOVA. End-of-train APD was computed using the average of beats 7 and 8 in each train. In addition, end-of-train APD after 3 minutes of pacing was compared to steady-state APD during fixed rate pacing at a cycle length of $400 \mathrm{~ms}$ using ANOVA. In all cases, a $\mathrm{P}<0.05$ was considered as significant.

Time constant values were obtained using a linear regression analysis on the natural logarithm of APD, thereby modeling the time course of APD change as a true exponential. APD values were normalized by subtracting the steady-state value and then a linear regression was performed on the natural logarithm of the APD for the first minute at the new stimulation rate. The slope of the regression line is then the inverse of the time constant of the exponential decay of the APD:

$$
\begin{gathered}
\mathrm{APD}=\operatorname{APD}(\mathrm{t})-\operatorname{APD}(\text { steady state }) \\
=\mathrm{APD}(\mathrm{t}=0) \cdot \mathrm{e}^{-\mathrm{t} / \tau} \\
\ln (\mathrm{APD})=(-1 / \tau) \mathrm{t}+\text { constant }
\end{gathered}
$$

In both pacing protocols (fixed pacing and pacing with an extrastimulus followed by a pause), 50 to 60 seconds worth of data points were used for the regression analysis immediately following the start of the corresponding protocol. Oscillation in APD was established whenever the variation in APD over 3 successive beats exceeded 2 standard deviations of our automated analysis system, ${ }^{12}$ or $5 \mathrm{~ms}$.
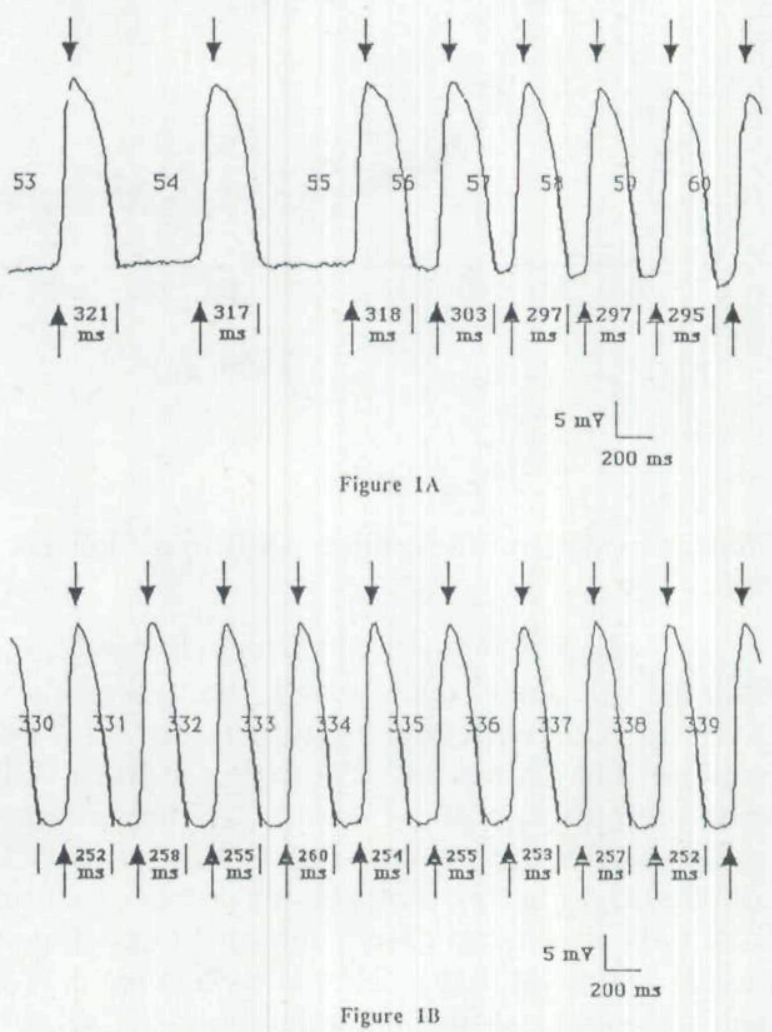

Figure 1. Digitized recordings of MAPs after a sudden shift in pacing cycle length from $1000 \mathrm{~ms}$ to $400 \mathrm{~ms}$ pacing cycle length (A) and after steady state at $400 \mathrm{~ms}$ (B). Vertical arrows under MAPs represent the computer detected locations of onset of activation. Vertical lines under MAPs represent intersection of tangent to phase 3 with baseline. Tangent lines to phase 3 superimposed on the action potentials by the program were used to estimate APD. Arrows on top of MAPs represent computer detected locations of peaks of MAPs. APD values are displayed under MAPs. The numbers in the center of each tracing refer to the MAP number within the data file. There is a decremental shortening of MAP during the first few beats at a pacing cycle length of $400 \mathrm{~ms}$ (A) and after several minutes a new steady state is reached (B). 


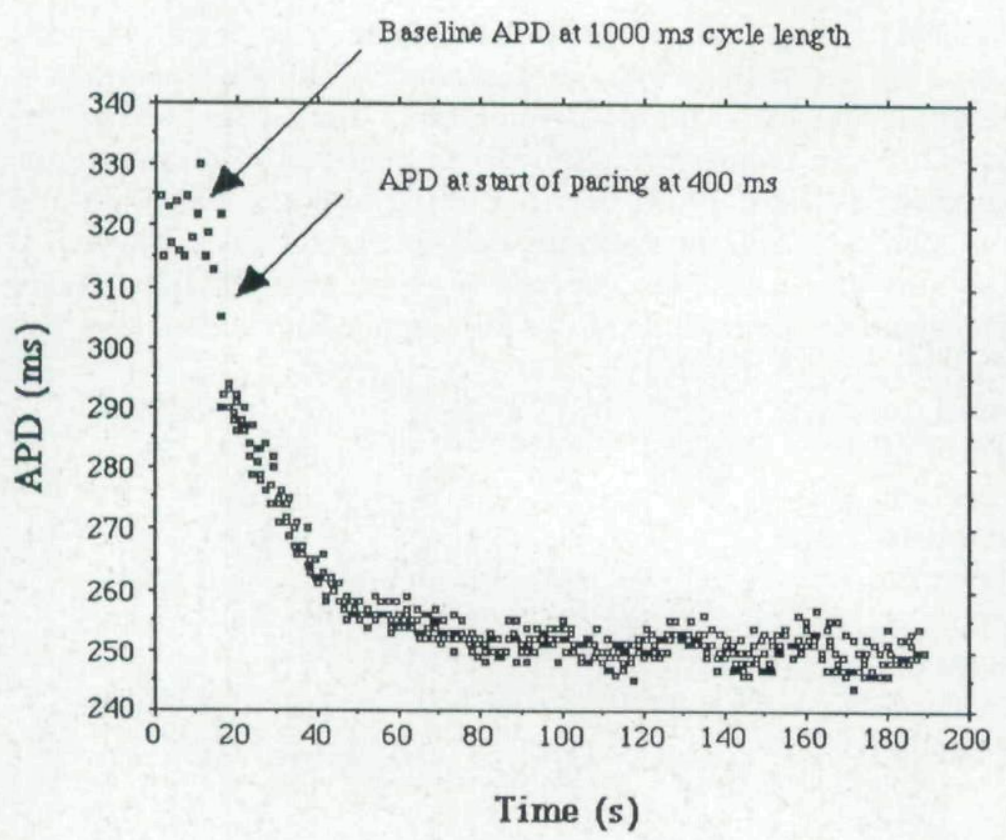

Figure 2. Time course of APD following a sudden shift in pacing cycle length from $1000 \mathrm{~ms}$ to $400 \mathrm{~ms}$. $A P D_{\mathrm{ss}}$ at $1000 \mathrm{~ms}$ is about $320 \mathrm{~ms}$. A sharp drop in APD is observed 20 to 30 seconds after the start of pacing at the new cycle length. After about 1 minute of pacing at $400 \mathrm{~ms}(\mathrm{~T}=80 \mathrm{~s})$, APD reaches a steady state of $252 \mathrm{~ms}$.

\section{Results}

\section{Change in APD with Sudden Shift to a Shorter PCL}

As expected, when the PCL was changed from $1000 \mathrm{~ms}$ to a faster cycle length, there was a decrease in APD with time. Figure 1 shows an example of the change in APD with a shift in PCL from $1000 \mathrm{~ms}$ to $400 \mathrm{~ms}$. Figure 1A shows endocardial MAPs during the shift to the faster PCL ( $400 \mathrm{~ms}$ ) and Figure 1B represents a train of action potentials after more than 2 minutes of pacing at a cycle length of $400 \mathrm{msec}$ when APD reached a steady state at the new cycle length.

A plot of APD versus time following a sudden shift to a PCL of $400 \mathrm{~ms}$ in another experiment is shown in Figure 2. At the start of pacing at the new frequency ( $400 \mathrm{~ms}$ ), there is a sharp drop in APD followed by a more gradual decrease until a steady state is reached after 80 seconds. APD change following a shift in PCL is summarized in Table I. As can be seen in Table I, as $\mathrm{PCL}_{\mathrm{f}}$ is shortened, there was a significant decrease in steadystate APD. Compared to APD at a cycle length of $1000 \mathrm{~ms}$, the percent drop in APD ranged from $9.5 \%$ at $600 \mathrm{~ms}$ to $26.4 \%$ at $300 \mathrm{~ms}$. APD during stimulation at $1000 \mathrm{~ms}$ did not change significantly over time. Figure 3 represents the steadystate APD at PCL $\mathrm{P}_{\mathrm{f}}$ of $300,400,500$, and $600 \mathrm{~ms}$.
The time course of decay of APD with time was characterized by two parameters, the time to reach steady state or $t_{s s}$, and the time constant of decay. The time to reach steady state, $t_{s s}$, was determined by finding the time interval from start of pacing at the fast cycle length to the time when the APD versus time curve is within 1 standard deviation from steady-state APD. ${ }^{12}$ Values for $t_{\mathrm{ss}}$ were $40.8 \pm 29.6$ seconds, $50.4 \pm 22.8$ seconds, $51.0 \pm 32.5$ seconds, and $52.8 \pm 14.8$ seconds for PCLs of $300,400,500$, and $600 \mathrm{~ms}$, respectively ( $\mathrm{P}=\mathrm{NS}$ for all comparisons $)$.

\section{Table I.}

$A P D_{s s}$ at $1000 \mathrm{~ms}$ and at Different $\mathrm{PCL}_{t}$ During Fixed Pacing

\begin{tabular}{|c|c|c|c|}
\hline $\begin{array}{l}\text { Pacing Cycle } \\
\text { Length } \\
\text { (ms) }\end{array}$ & $\begin{array}{c}\mathrm{APD}_{\mathrm{ss}} \text { at } \\
1000 \mathrm{~ms} \mathrm{PCL} \\
(\mathrm{ms})\end{array}$ & $\begin{array}{c}A P D_{s s} \text { at } \\
\text { PCL } \\
\text { (ms) }\end{array}$ & $\begin{array}{c}\text { Time } \\
\text { Constant } \\
\text { (s) }\end{array}$ \\
\hline 300 & $276 \pm 51$ & $203 \pm 31 \dagger^{*}$ & $14.9 \pm 0.8$ \\
\hline 400 & $274 \pm 28$ & $220 \pm 17+\S$ & $16.3 \pm 3.3$ \\
\hline 500 & $262 \pm 31$ & $229 \pm 21 \dagger \neq$ & $17.5 \pm 4.6$ \\
\hline 600 & $273 \pm 34$ & $247 \pm 29 \dagger$ & $20.3 \pm 4.79$ \\
\hline
\end{tabular}

t $\mathrm{P}<0.01$ with respect to baseline values

- $\mathrm{P}<0.05$ with respect to steady-state APD at $400 \mathrm{~ms}$

$\S \mathrm{P}<0.05$ with respect to steady-state APD at $500 \mathrm{~ms}$

$\ddagger P<0.05$ with respect to steady-state APD at $600 \mathrm{~ms}$

If $\mathrm{P}<0.01$ with respect to time constant at $300 \mathrm{~ms}$ pacing cycle length 


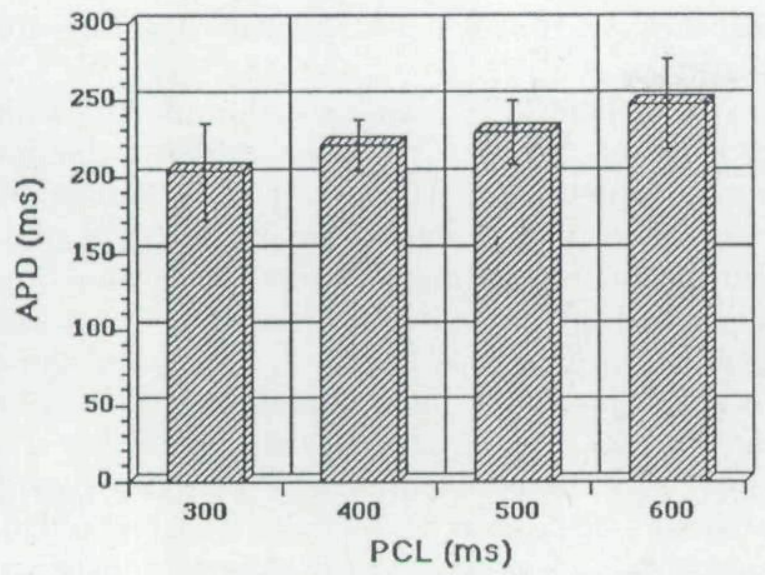

Figure 3. Steady-state APD following a shift from a pacing cycle length of $1000 \mathrm{~ms}$ to cycle lengths of 300 , 400,500 , and $600 \mathrm{~ms}$. APD at the fast cycle lengths ranged from $203 \mathrm{~ms}$ at $300 \mathrm{~ms}$ PCL to $247 \mathrm{~ms}$ at 600 ms PCL. See text for statistical comparisons of steadystate APD at different cycle lengths.

Figure 4 shows the technique used to deduce the time constant of decay of APD with time using an exponential curve fit. The linear curve fit of the natural logarithm of APD for the first 50 seconds at the new PCL provides an estimate of the time constant of decay, the slope of the line being the inverse of the time constant in seconds. As shown in Table I, the time constant decreased from 20.3 seconds at $600 \mathrm{~ms}$ cycle length to 16.3 seconds at $400 \mathrm{~ms}$ and 14.9 seconds at $300 \mathrm{~ms}(\mathrm{P}<0.01)$. The time constant value at $500 \mathrm{~ms}$ was $17.5 \mathrm{~ms}(\mathrm{P}=$
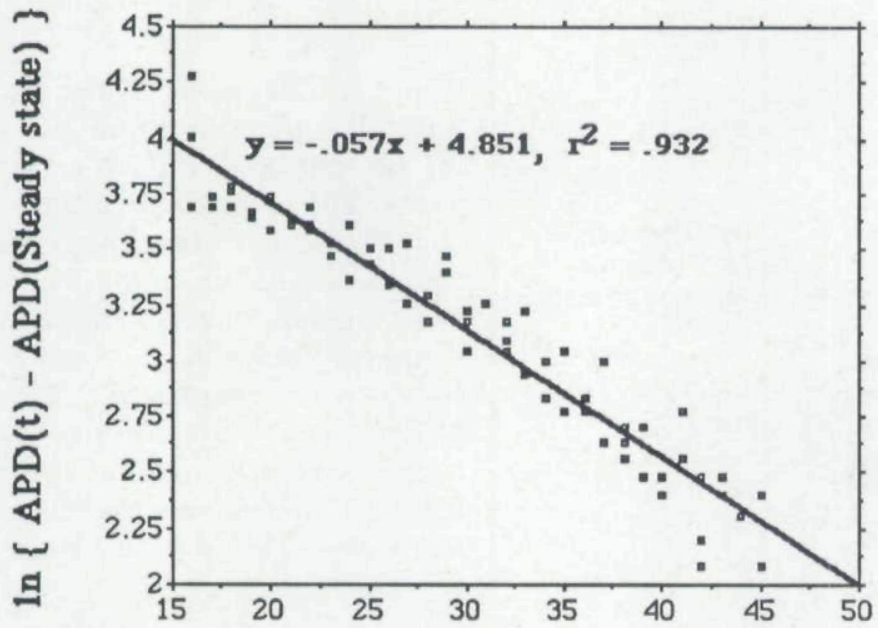

Time (s)
NS vs time constant at $600 \mathrm{~ms}$ cycle length). Regression values $\left(\mathrm{R}^{2}\right)$ ranged from 0.70 to 0.95 .

\section{Change in APD with Drive Train Pacing Incorporating an Inter-Train Pause}

Following a shift from fixed pacing at 1000 $\mathrm{ms}$ PCL to pacing at a cycle length of $400 \mathrm{~ms}$ with inter-train pause, APD decays progressively until reaching a new steady-state value. Figure 5 displays a digitized plot of MAPs at the end of pacing at $1000 \mathrm{~ms}$ and at the beginning of drive train pacing with a premature and a pause. Figure 6 is a plot of the change of APD with time using a 1second pause between trains. Time courses of basic drive, premature, and postpremature beats are shown.

With pause lengths of up to 8 seconds, APD progressively decreased with an increasing number of drive trains (Table II). Compared to APD of the first train, the percent drop in end-of-train APD after 3 minutes of pacing ranged from 10.1\% with a 1 -second inter-train pause to $2.1 \%$ with a 32-second pause. The change between APD of the first train and end-of-train APD after 3 minutes of pacing was not significant for an inter-train pause exceeding 8 seconds.

The time course of decrease in APD with successive drive trains is shown in Figure 7, which depicts a normalized plot of drive train APD for the first, second, tenth, as well as steady-state trains versus time. APD values were normalized

Figure 4. Linear curve fit of the natural logarithm of APD versus time following a sudden switch in pacing cycle length. Data points during the first 50 seconds after the shift in pacing frequency were used. APD was normalized by subtracting the steady-state value. The time constant in seconds is the negative inverse of the slope of the line (here 1/ 0.057 or $17.5 \mathrm{~s}$ ). 


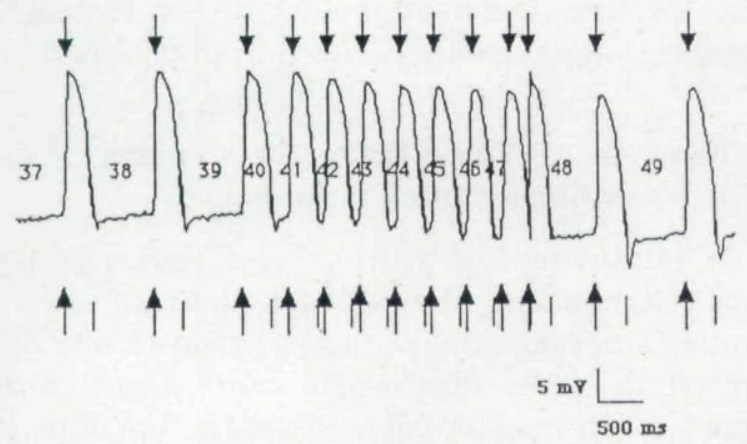

Figure 5. Digitized recordings of MAPs at the end of pacing at $1000 \mathrm{~ms}$ and beginning of drive train pacing with an extrastimulus and a pause. Drive train pacing starts with beat 39 and beat 47 is the response to the premature stimulus. As in Figure 1, tangent lines in phase 3 were superimposed on the MAPs to provide an estimate for APD. Arrows and vertical lines are as in Figure 1. Note that onset of extrastimulus (\#47) occurs before the intersection of the tangent line to beat 46 but the tangent method allows for the calculation of an APD.

with respect to APD of the first drive train for each individual cycle length. As with fixed rate pacing, the time course of decay of APD was characterized by a time constant and a time to reach steady state. Values for $\mathrm{t}_{\mathrm{ss}}$ increased from $50.4 \pm 22.8$ seconds with no inter-train pause to $50.8 \pm 16.8$ seconds, $55.9 \pm 13.4$ seconds, and $56.2 \pm 14.6$ seconds with pauses of 1 second, 2 seconds, and 4 seconds, respectively. However, comparisons did not reach significance.

The technique used to determine the time constant of decay of APD was similar to the one used in the case of fixed rate pacing. Time constant values were longer at greater pause lengths. During pacing without a pause, the mean time constant at a PCL of $400 \mathrm{~ms}$ was $16.3 \pm 3.3 \mathrm{sec}-$ onds. With pauses of 1 second, 2 seconds, and 4 seconds the mean time constants were $21.0 \pm 7.6$ seconds, $25.3 \pm 4.5$ seconds and $28.0 \pm 9.9$ seconds, respectively. Differences between pacing without a pause and pacing with 2-second and 4second pauses as well as the difference between 1-second and 4-second pauses were significant at the 0.05 level. Time constants for pause lengths exceeding 4 seconds were not computed because of the small number of data points owing to the large pause.

\section{Oscillations in APD}

Damped oscillation in APD with fixed rate pacing and following a sudden shift to a faster cycle length occurred in 4 out of 29 cases (13.8\%). The occurrence rate of oscillation was higher with the fastest cycle lengths $(60 \%$ with a shift to 300 ms PCL, $11 \%$ with $400 \mathrm{~ms}$ PCL, and $2 \%$ with 500 and $600 \mathrm{~ms}$ PCL). Persistent oscillations were not observed.

During drive train pacing with inter-train

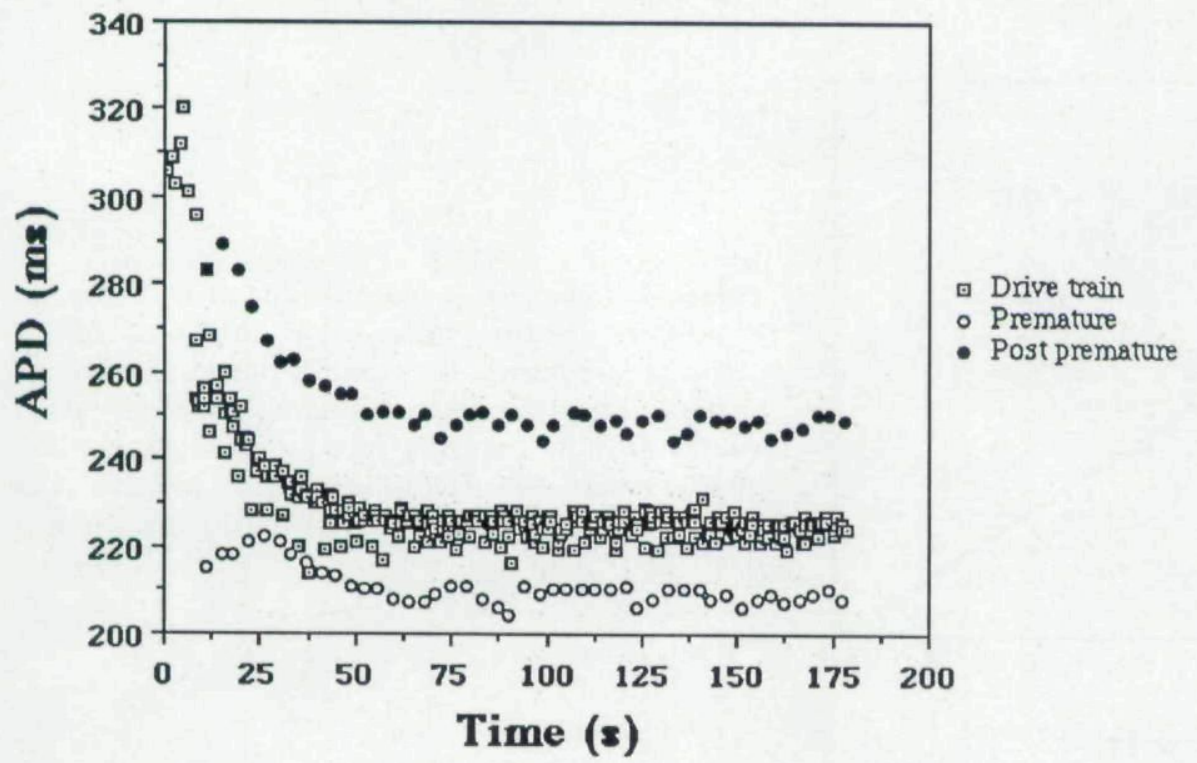

Figure 6. Change of APD with time following a shift from pacing at $1000 \mathrm{~ms}$ to drive train pacing with extrastimulus and $a$ pause between trains. The time course of APD of end-of-train beats, premature beat and postpremature beat with a 1-s intertrain pause are shown $(n=500$ MAPs). Note the similarity in time course of change of APD between the beats in the drive train and the postpremature beats. 


\section{Table II.}

End of Train APD of the First Drive Train and after 3 Minutes of Drive Train Pacing Incorporating an Inter-Train Pause

\begin{tabular}{cccc}
\hline $\begin{array}{c}\text { Pause } \\
\text { Length } \\
\text { (s) }\end{array}$ & $\begin{array}{c}\text { End of Train APD } \\
\text { of First Drive } \\
\text { Train } \\
(\mathbf{m s})\end{array}$ & $\begin{array}{c}\text { End of Train APD } \\
\text { after } 3 \text { Minutes } \\
\text { of Pacing } \\
\text { (ms) }\end{array}$ & $\begin{array}{c}\text { Percent } \\
\text { Drop }\end{array}$ \\
\hline 1 & $261 \pm 25$ & $235 \pm 21 \dagger \ddagger$ & $10.1 \pm 3.4$ \\
2 & $270 \pm 28$ & $248 \pm 23 \dagger$ & $8.2 \pm 3.2$ \\
4 & $266 \pm 22$ & $245 \pm 21 \dagger$ & $7.9 \pm 3.9$ \\
8 & $264 \pm 24$ & $252 \pm 24 \dagger$ & $4.6 \pm 1.8$ \\
16 & $268 \pm 31$ & $260 \pm 26$ & $3.0 \pm 1.8$ \\
32 & $261 \pm 34$ & $255 \pm 29$ & $2.1 \pm 2.3$ \\
\hline
\end{tabular}

$\dagger \mathrm{P}<0.01$ with respect to first train values

$\ddagger P<0.05$ with respect to pause lengths of $2,4,8,16$, and $32 \mathrm{~s}$

pause, oscillations of APD within each drive train was observed in all 12 experiments. Figure 8 shows the APD of all 8 beats of a drive train taken from one experiment with an inter-train pause length of 1 second and demonstrates oscillation of APD within the drive train. The percent of drive trains exhibiting oscillation ranged from $3 \%$ to $27 \%$ (mean $12 \pm 6 \%$ ). The occurrence rate of oscillation was $9 \%$ with a 1 -second pause, $17 \%$ with pause lengths of 2 and 4 seconds, $11 \%$ with an 8 -

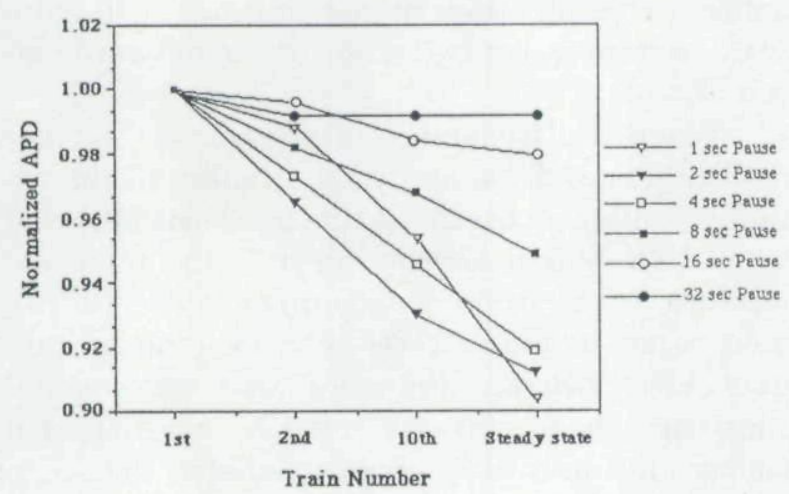

Figure 7. Change of APD with time following a shift from pacing at $1000 \mathrm{~ms}$ to drive train pacing with extrastimulus and a pause between trains. Normalized APD values of first, second, tenth, and steady-state trains with different pause lengths are shown. Note that the drop in APD is $<2 \%$ for pause lengths equal to or exceeding 16 seconds.

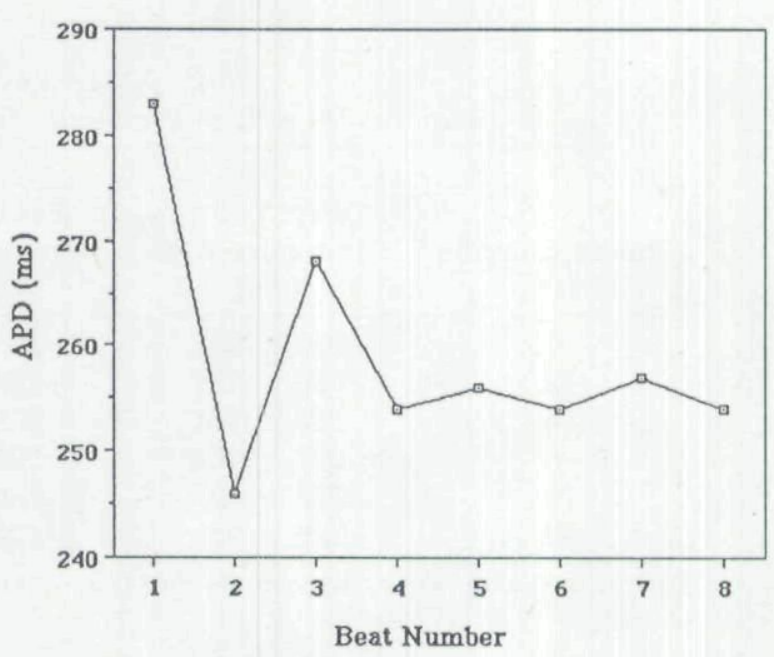

Figure 8. Example of damped oscillations in drive train APD (8 $\mathrm{S}_{1}$ at $400 \mathrm{~ms}$ ) following pacing at $1000 \mathrm{~ms}$ observed in one experiment. Inter-train pause length was 1 second. This pattern, although infrequent, was sustained over the next several drive trains when it did occur.

second pause, $8 \%$ with a 16 -second pause and $6 \%$ with a 32-second pause. However, none of these differences was significant. No attempt was made to control the diastolic interval in order to systematically correlate it with the occurrence of oscillation. Within the observed range of coupling intervals of the first beats in the drive trains, there was no correlation between the diastolic interval preceding those beats and the onset of oscillation in APD. However, there was not a wide enough range of observed coupling intervals to draw any definitive conclusions. It was interesting to note that, when oscillation did occur during one drive train, it was sustained over the next several trains with almost identical patterns of oscillation. The amplitude of oscillation ranged from $8 \mathrm{~ms}$ to 15 ms (mean $12 \pm 2 \mathrm{~ms}$ ).

\section{Variation in APD of Premature Beats}

Changes in APD of the premature beats (Table III) behaved in a similar fashion to APD of the drive train beats. Table III shows the change in the APD of the premature beats with inter-train pause length. APD of premature beats dropped significantly with an increase in the number of drive trains for inter-train pause lengths of 1,2, 4, and 
Table III.

Variation in APD of Premature Responses with Inter-Train Pause Length

\begin{tabular}{ccccc}
\hline $\begin{array}{c}\text { Pause Length } \\
(\mathbf{s})\end{array}$ & $\begin{array}{c}\text { First Train } \\
\text { Premature APD } \\
(\mathbf{m s})\end{array}$ & $\begin{array}{c}\text { Second Train } \\
\text { Premature APD } \\
(\mathbf{m s})\end{array}$ & $\begin{array}{c}\text { Tenth Train } \\
\text { Premature APD } \\
(\mathbf{m s})\end{array}$ & $\begin{array}{c}\text { Premature APD } \\
\text { after } \mathbf{3} \text { Minutes } \\
\text { of Pacing } \\
(\mathbf{m s})\end{array}$ \\
\hline 1 & $230 \pm 25 \dagger$ & $226 \pm 21 \dagger$ & $219 \pm 20 \dagger$ & $208 \pm 16$ \\
2 & $230 \pm 23 \dagger$ & $229 \pm 21$ & $225 \pm 19$ & $222 \pm 14$ \\
4 & $233 \pm 16 \dagger$ & $230 \pm 17 \dagger$ & $226 \pm 14$ & $222 \pm 13$ \\
8 & $231 \pm 15 \dagger$ & $228 \pm 16$ & $225 \pm 14$ & $223 \pm 15$ \\
16 & $227 \pm 18$ & $230 \pm 14$ & $224 \pm 15$ & $224 \pm 15$ \\
32 & $226 \pm 20$ & $222 \pm 17$ & $223 \pm 20$ & $223 \pm 20$ \\
\hline
\end{tabular}

$\dagger P<0.05$ with respect to premature APD after 3 minutes of pacing

8 seconds $(\mathrm{P}<0.05$ between premature in the first train and that after 3 minutes of pacing). Comparisons were not significant at pause lengths equal to or exceeding 16 seconds.

\section{Comparison of Change in APD During Pacing With and Without Pause}

In order to determine the effect of the introduction of a pause on the time course of APD, we compared the steady-state value at a fixed PCL of $400 \mathrm{~ms}$ (protocol A) to that obtained with protocol B $\left(8 \mathrm{~S}_{1}\right.$ at $400 \mathrm{~ms}, \mathrm{~S}_{2}$, followed by a pause). Steadystate APD at $400 \mathrm{~ms}$ PCL was $215 \pm 11 \mathrm{~ms}$, whereas end-of-train APD with pauses after 3 minutes of pacing increased from $223 \pm 17 \mathrm{~ms}$ with 1-second pause to $255 \pm 29 \mathrm{~ms}$ with 32 -second pause. Steady-state APD with constant cycle length pacing was significantly smaller than that at each of the six different pause lengths used (P $<0.05$ ).

\section{Discussion}

The major findings of this study are that with a sudden decrease in PCL (as might be seen with "burst pacing" during an electrophysiology study), APD shortens until a new steady state is reached. As $\mathrm{PCL}_{\mathrm{f}}$ is shortened, steady-state APD is shorter and interestingly the time constant of change is also shorter. When pacing using an inter-train pause (as might be seen during programmed electrical stimulation in an electrophysiology study), end-of-train APD after 3 minutes of pacing is always longer than during pacing at a constant cycle length and APD shortens with successive drive trains using inter-train pause lengths of 8 seconds or less. With both pacing protocols, 40 to 60 seconds are required to reach a new steady state.

Rate dependent changes of the action potential. Several investigators have demonstrated a significant shortening of the action potential in response to increased heart rate. ${ }^{1,16-19}$ This shortening with an increased rate of stimulation has been associated with an increase in peak tension $^{20,21}$ and is thought to be partially caused by an elevated intracellular $\left(\mathrm{Ca}^{2+}\right)_{\mathrm{i}}$. Such an increase in intracellular calcium could shorten the action potential either by decreasing the slow inward current $\left(\mathrm{I}_{\mathrm{si}}\right)$ or by increasing an outward current. $^{22}$

When the frequency of stimulation is suddenly increased, an abrupt shortening of the action potential occurs in the first few beats followed by a decremental shortening over the next few minutes. ${ }^{23}$ The initial shortening has been attributed to an incomplete recovery of plateau conductances, whereas the slow and decremental shortening has been attributed to an increased background outward current mediated by an increased $\mathrm{Na} / \mathrm{K}$ pump activity. ${ }^{23}$ Bassingthwaighte et al. ${ }^{24}$ reported an increase in outward background current following an increase in the rate of voltage-clamp stimuli, attributing the change to an increase in potassium conductance as a consequence of a rise in intracellular calcium. It would therefore be expected, as has been previ- 
ously shown (Table I), that the effect of fast pacing on the cardiac membrane would be more pronounced at the shorter cycle lengths, due to an increased outward current. Similar results for endocardial MAPs in vivo were seen in this study. When comparing the steady-state APD values at all four pacing cycle lengths $(600,500,400$, and $300 \mathrm{~ms}$ ), there was a significant shortening of the steady-state APD as the cycle length was decreased, as can be seen in Table I.

Time constant of decay of APD with increased rate. In addition to a difference in steady-state APD at different PCLs, we found that the time constant of change of the new APD was shorter with a shorter PCL. The time to reach steady-state APD varied in a way that was similar to the time constant but the differences did not reach significance. Other investigators have examined the adaptation of APD to a new PCL but have not compared a change to different $\mathrm{PCL}_{\mathrm{f}}$. Janse et al. ${ }^{25}$ showed that after a sudden increase or decrease in pacing frequency, a steady-state value of the duration of refractoriness is reached after several hundred beats. Carmeliet ${ }^{26}$ found that a steadystate value for the transmembrane action potential is established after 40 to 50 beats at the new pacing rate. Seed et al., ${ }^{4}$ while summarizing the results of electrophysiology studies on 14 patients, reported that at least 3 minutes were required for a new steady-state APD to be reached following a switch to a new pacing frequency. In this study, the change in APD after a shift to $\mathrm{PCL}_{\mathrm{f}}$ was modeled as a single exponential. Both the time constants and the time to reach steady state were substantially shorter than in Boyett's study. ${ }^{23}$ This difference may reflect a difference in the tissues (dog Purkinje vs endocardial muscle fiber) as well as a difference in the technique used to calculate time constants.

The explanation for the shorter time constants at shorter $\mathrm{PCL}_{\mathrm{f}}$ is not completely clear. One potential explanation is that as the pacing rate is increased, the diastolic interval is decreased. As a result, the average time that the cells spend in a depolarized state is increased, which leads to an increased calcium loading. An increase in cytoplasmic calcium causes an increased rate of calcium inactivation which causes a shortening of the action potential. The faster the new pacing rate, the greater the calcium loading and hence the faster the rate of shortening of the action potential. Alternatively, calcium loading may cause an increase in the component of transient outward current $\mathrm{I}_{\text {to }}$ which is calcium activated. An increase in $\mathrm{I}_{\text {to }}$ causes a reduction in APD. ${ }^{27,28}$ However, it has been shown that $\mathrm{I}_{\text {to }}$ is less prominent in the endocardium compared to the epicardium. ${ }^{27,28}$ Since action potentials in this study were recorded from the endocardium, it is less likely that $I_{\text {to }}$ will have a major effect on APD.

$A P D$ during pacing using an inter-train pause. Programmed stimulation using a PCL faster than the spontaneous cycle length and incorporating an inter-train pause is often used in both clinical and experimental electrophysiology studies to determine refractory periods and attempt to induce tachyarrhythmias. Changes in action potential using drive train pacing with an inter-train pause have not been carefully examined. However, Morady et al. ${ }^{29}$ showed in a recent study that the cumulative effect of drive train pacing with inter-train pause on the ventricular effective refractory period (VERP) persisted until the pause length was at least 20 seconds. In addition, with a short inter-train pause, APD may not reach the baseline value it had prior to drive train pacing. Hence, drive trains may result in a cumulative effect on refractoriness leading to gradual shortening of VERP. Our experiments on endocardial MAPs showed similar results. Not surprisingly, when comparing fixed rate ventricular pacing to drive train pacing incorporating an inter-train pause, APD was always shorter during constant rate pacing regardless of the magnitude of the inter-train pause. With inter-train pauses shorter than 16 seconds, there was a decremental shortening in APD in successive drive trains until between 50 and 56 seconds (depending on the intertrain pause) at which time a steady state was reached. With inter-train pauses of 16 and $32 \mathrm{~s}$, there was no change in APD in successive drive trains. This is likely because the length of the inter-train pause was adequate to prevent any cumulative effect on APD of the prior drive train.

Oscillation in action potential duration during drive train pacing. Alternation in APD following a sudden shift to a faster pacing rate has been reported by several investigators..$^{5,18,30}$ This alternation has been attributed to the change in degree of recovery of the membrane between consecutive 
stimuli. The first beat at the fast rate is preceded by a shorter diastolic interval that leads to a shorter refractory period and a shorter APD. Since pacing is periodic, the second beat at the fast rate is thus preceded by a longer diastolic interval than the first beat, and the refractory period as well as APD are consequently longer. This process repeats itself with the following beats, leading to an oscillation in APD and refractory period. In our experiments, oscillation in APD was observed in at least one drive train in all experiments with a varying degree of occurrence. Oscillation was noted in $17 \%$ of drive train APDs with inter-train pause lengths of 2 and 4 seconds. The occurrence of oscillation dropped as pause length increased to 32 seconds, but the differences were not significant. In the present study, no attempt was made to control the diastolic interval in order to correlate it with the occurrence of oscillations. ElHarrar and Surawicz ${ }^{31}$ reported that the coupling interval of the first beat at the fast rate must be < $400 \mathrm{~ms}$ in order to produce oscillation in APD. With drive train pacing at 400-ms cycle length, oscillation was only infrequently observed in our experiments. The factors responsible for the sporadic occurrence of oscillation in APD during pacing incorporating an inter-train pause remain unclear.

Limitations to the current study. Although the magnitude of APDs obtained in this study were characteristic of those of ventricular muscle rather than Purkinje fibers, a contribution of Purkinje fibers or transitional cells to the MAP signal obtained in this study cannot be excluded. However, the MAP signal we obtained likely represents a true sum of endocardial electrophysiological activity in vivo. In addition, only a single ventricular site was examined in each study and differences between endocardial and epicardial action potentials or different ventricular sites cannot be commented upon. Another limitation of the present study is that only a single baseline drive cycle length $(1000 \mathrm{~ms})$ and a single drive train cycle length (400 ms) were used. The effects of different baseline and drive train cycle lengths cannot be determined from this study. Also, the time constants presented for changes in refractoriness may bear no relationship to the time constants that occur in man.

Implications for clinical electrophysiological studies. In order to study the recovery of excitability of cardiac tissue or to attempt to induce reentrant arrhythmias in the electrophysiology laboratory, it is customary to use a pacing protocol consisting of a train of 8 driving stimuli at cycle lengths ranging from 350 to $600 \mathrm{~ms}$ followed by one or more extrastimuli scanning diastole. Although several drive trains often occur prior to reaching refractoriness during scanning of diastole using a decremental method, the number of drive trains that are actually used is variable. These drive trains represent a de facto conditioning period prior to reaching refractoriness. In this study, using a similar pacing protocol and intertrain pause lengths $<16$ seconds, APD did not reach a steady state during successive drive trains until a mean of approximately 1 minute in the study. However, in some experiments, up to 2 minutes were required to reach steady state. This suggests that to achieve steady state during programmed stimulation and produce consistent results in the determination of cardiac muscle refractoriness and potentially in the induction of tachyarrhythmias, the conditioning period routinely applied prior to the introduction of the premature(s) should be at least 2 minutes.

\section{References}

1. Gibbs C, Johnson E. Effect of changes in frequency of stimulation upon rabbit ventricular action potential. Circ Res 1981; 9:165-170.

2. Denes P, Wu D, Dhingra R, et al. The effects of cycle length on cardiac refractory periods in man. Circulation 1974; 49:32-41.

3. Guss S, Kastor J, Josephson M, et al. Human ventricular refractoriness. Circulation 1976; 53:450456.

4. Seed W, Noble M, Oldershaw P, et al. Relation of human cardiac action potential duration to the interval between beats: Implications for the validity of rate corrected QT interval $\left(\mathrm{QT}_{\mathrm{c}}\right)$. Br Heart J 1987; 57:32-37.

5. Vick R. Action potential duration in canine Purkinje tissue: Effects of preceding excitation. J Electrocardiol 1971; 4(2):105-115.

6. Franz M. Long-term recording of monophasic action potentials from human endocardium. Am J Cardiol 1983; 51:1629-1634.

7. Franz M, Bargheer K, Raffenbeul W, et al. Monophasic action potential mapping in human subjects with normal electrocardiograms: Direct evidence of the genesis of the $\mathrm{T}$ wave. Circulation 1987; 75(2):379-386.

8. Franz M, Swerdlow C, Bing Liem L, et al. Cycle 
length dependence of human action potential duration in vivo. J Clin Invest 1988; 82(3):972-979.

9. Olsson S, Brorson L, Edvardsson N, et al. Estimation of ventricular repolarization in man by monophasic action potential technique. Eur Heart J 1985; 6(Suppl. D):71-79.

10. Takeshi I, Karagueuzian H, Kichol H, et al. Relation of monophasic action potential recorded with contact electrode to underlying transmembrane action potential properties in isolated cardiac tissues: A systematic microelectrode validation study. Cardiovasc Res 1988; 22:255-264.

11. Franz M, Burkhoff D, Spurgeon $\mathrm{H}$, et al. In vitro validation of a new cardiac catheter technique for recording monophasic action potentials. Eur Heart J 1986; 7:34-41.

12. Kanaan N, Jenkins I, Kadish A. An automatic microcomputer system for analysis of monophasic action potentials. PACE 1990; 13:196-206.

13. Damiano B, Rosen M. Effects of pacing on triggered activity induced by early afterdepolarizations. Circulation 1984; 69(5):1013-1025.

14. Ben David J, Zipes D. Differential response to right and left ansae subclaviae stimulation of early afterdepolarizations and ventricular tachycardia induced by cesium in dogs. Circulation 1988; 78(5):1241-1250.

15. Bailie D, Inoue $H$, Kaseda $S$, et al. Magnesium suppression of early afterdepolarizations and ventricular tachyarrhythmias induced by cesium in dogs. Circulation 1988; 77(6):1395-1402.

16. Hoffman B, Suckling E. Effect of heart rate on cardiac membrane potentials and the unipolar electrogram. Am J Physiol 1954; 179:123-130.

17. Miller J, Wallace A, Freezor M. A quantitative comparison of the relation between the shape of the action potential and the pattern of stimulation in canine ventricular muscle and Purkinje fibers. J Mol Cell Cardiol 1971; 2:3-19.

18. Boyett M, Jewell B. A study of the factors responsible for rate-dependent shortening of the action potential in mammalian ventricular muscle. J Physiol 1978; 285:359-380.

19. Gettes L, Morehouse N, Surawicz B. Effect of premature depolarization on the duration of action potentials in Purkinje and ventricular fibers of the moderator band of the pig heart. Role of proximity and the duration of the preceding action potential. Circ Res 1972; 30:55-66.
20. Braveny P, Sumbera J. Interrelations between action potential and contractile response of heart muscle. Cardiovasc Res 1971; 1(Suppl.):109-111.

21. Allen D. On the relationship between action potential duration and tension in cat papillary muscle. Cardiovasc Res 1977; 11:210-218.

22. Boyett M, Jewell B. Analysis of the effects of changes in rate and rhythm upon electrical activity in the heart. Prog Biophysiol Mol Biol 1980; 36:152.

23. Boyett M, Fedida D. Changes in the electrical activity of dog cardiac Purkinje fibres at high heart rates. J Physiol 1984; 350:361-391.

24. Bassingthwaighte J, Fry C, McGuigan J. Relationship between internal calcium and outward current in mammalian ventricular muscle: A mechanism for the control of the action potential duration. J Physiol 1976; 262:15-37.

25. Janse M, van der Steen D, van Dam R, et al. Refractory period of the dog's ventricular myocardium following sudden changes in frequency. Circ Res 1969; 24:251-262.

26. Carmeliet E. Influence du rhythme sur la duree du potentiel d'action ventriculaire cardiaque. Arch Intern Physiol 1955; 63:222.

27. Litovsky SH, Antzelevitch C. Rate dependence of action potential duration and refractoriness in canine ventricular endocardium differs from that of epicardium: Role of the transient outward current. J Am Coll Cardiol 1989; 14(4):1053-1066.

28. Litovsky SH, Antzelevitch C. Transient outward current prominent in canine ventricular epicardium but not endocardium. Circ Res 1988; 62(1):116-126.

29. Morady F, Kadish A, Rosenheck S, et al. Effect of the inter-train pause on the ventricular effective refractory period measured by the extrastimulus technique. PACE 1990; 13:405-409.

30. Tchou P, Lehmann M, Dongas J, et al. Effect of sudden rate acceleration on the human His-Purkinje system: Adaptation of refractoriness in a damped oscillatory pattern. Circulation 1986; 73(5):920-929.

31. El-Harrar V, Surawicz B. Cycle length effect on restitution of APD in dog cardiac fibers. Am J Physiol 1983; 244:H782. 
Copyright of Pacing \& Clinical Electrophysiology is the property of Blackwell Publishing Limited and its content may not be copied or emailed to multiple sites or posted to a listserv without the copyright holder's express written permission. However, users may print, download, or email articles for individual use. 